\title{
Delay-dependent exponential stabilization of positive 2D switched state-delayed systems in the Roesser model
}

\author{
Zhaoxia Duan ${ }^{\text {a }}$, Zhengrong Xiang ${ }^{\text {a,* }}$, Hamid Reza Karimi ${ }^{\text {b }}$ \\ a School of Automation, Nanjing University of Science and Technology, Nanjing 210094, People's Republic of China \\ ${ }^{\mathrm{b}}$ Department of Engineering, Faculty of Engineering and Science, University of Agder, N-4898 Grimstad, Norway
}

A B S T R A C T

This paper deals with the controller synthesis for a class of positive two-dimensional (2D) switched delay systems described by the Roesser model. This kind of systems has the property that the states take nonnegative values whenever the initial boundaries are nonnegative, some delay-dependent sufficient conditions for the exponential stability of positive 2D switched systems with state delays are given. Furthermore, the design of positive state feedback controller under which the resulting closed-loop system meets the requirements of positivity and exponential stability is presented in terms of linear matrix inequalities (LMIs). An example is included to illustrate the effectiveness of the proposed approach.

\section{Introduction}

Two-dimensional (2D) systems exist in many practical applications, such as circuit analysis, digital image processing, signal filtering and thermal power engineering $[8,19,48]$. Thus the analysis and synthesis of $2 \mathrm{D}$ systems are interesting and challenging problems, and have received considerable attention, for example, 2D state-space realization theory was researched in [14], the stability and 2D optimal control theory were studied in [7,12,17], and the $H_{\infty}$ filtering problem for 2D Markovian jump systems was addressed in [42].

The most popular models of 2D linear systems were introduced by Roesser, Fornasini and Marchesini [14,15] and Kurek [27]. These models have been extended for positive systems in [20,21]. A positive system means that its states and outputs are nonnegative whenever the initial conditions and inputs are nonnegative [13,21]. Positive 2D systems are needed in many cases such as the wave equation in fluid dynamics, the Poisson's equation, and the heat equation which describes the temperature (using thermodynamic temperature scale) in a given region over time. These facts stimulate the research on positive 2D discrete-time systems. The choice of the forms of Lyapunov functions for positive 2D Roesser model was investigated in [23]. The problem of stability analysis for positive 2D fractional systems was investigated in [24]. Furthermore, the reaction of real world systems to exogenous signals is never instantaneous and always infected by time delays [16,36-38]. The reachability, minimum energy control and realization problem for positive 2D discrete-time systems with delays was analyzed in [22]. And the stability analysis for positive 2D delayed systems was investigated in [3,25,26].

On the other hand, a considerable interest has been devoted to the research of switched systems during the recent decades. A switched system comprises a family of subsystems described by continuous or discrete-time dynamics, and a 
switching law that specifies the active subsystem at each instant of time. Apart from the switching strategy to improve control performance [10,35], switched systems also arise in many engineering applications [2,6]. Many techniques are effective tools dealing with switched systems, such as common quadratic Lyapunov function method, multiple Lyapunov function method, and average dwell time approach [1,9,28-33,39-41,49]. Recently, [34,45] studied the model reduction for linear switched systems, and $[43,46]$ focused on the problems of stability and control synthesis by using sliding mode control method.

In addition, it is well known that the switching phenomenon may also occur in practical 2D systems, so 2D switched systems have also attracted considerable research attention. There are a few reports on 2D discrete switched systems, Benzaouia et al. firstly considered 2D switched systems with arbitrary switching sequences in [4] and investigated the stabilizability problem of 2D switched systems in [5], the generalized $H_{2}$ fault detection for 2D Markovian jump systems was studied in [44]. Recently, the exponential stabilization of 2D switched Roesser model was firstly investigated in [47]. For positive 2D switched systems, a typical physical application is the thermal process with multiple modes. The positive value of temperature (using thermodynamic temperature scale) depends on position variable, time variable and the heating intensity (switching among multiple modes), so this system can be modeled as a positive 2D switched system. By using algebraic techniques, sufficient and necessary conditions were first provided for the asymptotic stability of positive 2D switched systems described by the Roesser models in [11]. However, to the best of our knowledge, there has been little literature considering the control problem of positive 2D switched systems with time delays, which motivates the present work.

In this paper we will investigate the problems of delay-dependent stability analysis and stabilization for positive 2D switched linear systems with delays. The main contributions of this paper lie in: (1) By constructing an appropriate co-positive Lyapunov function, we first analyze the delay-dependent exponential stability of positive 2D switched Roesser model with state delays. (2) Instead of using algebraic techniques [11] which have been employed for the analysis of positive 2D switched systems, the average dwell time approach is applied to our developments which are based on LMIs. (3) Based on the well established results of exponential stability analysis, equivalent conditions in terms of LMIs are obtained for the existence of stabilizing positive state feedback controllers. A remarkable advantage of these conditions lies in the easy verification by using some standard numerical software.

The remainder of the paper is organized as follows. In Section 2, problem statement and some definitions concerning the positive 2D switched discrete linear systems with delays are given. In Section 3, some results concerning the delaydependent exponential stability and stabilization of positive 2D switched linear systems are presented. In Section 4, a physical example is given to illustrate the effectiveness of the proposed approach. Finally, concluding remarks are provided in Section 5. The following notation will be used.

Notations: In this paper, the superscript "T" denotes the transpose. The notation $X>Y(X \geqslant Y)$ means that matrix $X-Y$ is positive definite (positive semi-definite, respectively). $A \succeq 0(\preceq 0)$ means that all entries of matrix $A$ are nonnegative (non-positive). $A \succ 0(\prec 0)$ means that all entries of matrix $A$ are positive (negative). $R^{n \times m}$ denotes the set of $n \times m$ real matrices. The set of real $n \times m$ matrices with nonnegative entries will be denoted by $R_{+}^{n \times m}$ and the set of nonnegative integers will be denoted by $Z_{+}$. $R_{+}^{n}$ denotes the set of vectors with nonnegative entries. The $n \times n$ identity matrix will be denoted by $I_{n}$.

\section{Problem formulation and preliminaries}

Consider the following 2D switched Roesser model with state delays:

$$
\left[\begin{array}{l}
x^{h}(i+1, j) \\
x^{v}(i, j+1)
\end{array}\right]=A^{\sigma(i, j)}\left[\begin{array}{l}
x^{h}(i, j) \\
x^{v}(i, j)
\end{array}\right]+A_{d}^{\sigma(i, j)}\left[\begin{array}{l}
x^{h}\left(i-d_{h}(i), j\right) \\
x^{v}\left(i, j-d_{v}(j)\right)
\end{array}\right]+B^{\sigma(i, j)} u(i, j),
$$

where $i$ and $j$ are integers in $Z_{+}, x^{h}(i, j)$ is the horizontal state in $R^{n_{1}}, x^{v}(i, j)$ is the vertical state in $R^{n_{2}}, x(i, j)$ is the whole state in $R^{n} . \sigma(\cdot): Z_{+} \times Z_{+} \rightarrow \underline{N}=\{1,2, \ldots, N\}$ is the switching signal. $N$ denotes the number of subsystems. $A^{k}, A_{d}^{k}$ and $B^{k}, k \in \underline{N}$, are constant matrices with appropriate dimensions and can be represented as

$$
A^{k}=\left[\begin{array}{ll}
A_{11}^{k} & A_{12}^{k} \\
A_{21}^{k} & A_{22}^{k}
\end{array}\right], \quad A_{d}^{k}=\left[\begin{array}{ll}
A_{d 11}^{k} & A_{d 12}^{k} \\
A_{d 21}^{k} & A_{d 22}^{k}
\end{array}\right], \quad B^{k}=\left[\begin{array}{c}
B_{1}^{k} \\
B_{2}^{k}
\end{array}\right],
$$

$d_{h}(i)$ and $d_{l}(j)$ are delays along horizontal and vertical directions, respectively. We assume that $d_{h}(i)$ and $d_{\imath}(j)$ satisfy

$$
d_{h L} \leqslant d_{h}(i) \leqslant d_{h H}, \quad d_{v L} \leqslant d_{v}(j) \leqslant d_{v H},
$$

where $d_{h L}, d_{h H}$ and $d_{v L}, d_{v H}$ denote the lower and upper delay bounds along horizontal and vertical directions, respectively. The boundary conditions are given by

$$
\begin{array}{lll}
x^{h}(i, j)=h_{i j}, & \forall 0 \leqslant j \leqslant z_{1}, & -d_{h H} \leqslant i \leqslant 0 \\
x^{h}(i, j)=0, & \forall j>z_{1}, & -d_{h H} \leqslant i \leqslant 0 \\
x^{v}(i, j)=v_{i j}, & \forall 0 \leqslant i \leqslant z_{2}, & -d_{v H} \leqslant j \leqslant 0 \\
x^{v}(i, j)=0, & \forall i>z_{2}, & -d_{v H} \leqslant j \leqslant 0, \\
h_{00}=v_{00} & &
\end{array}
$$


where $z_{1}<\infty$ and $z_{2}<\infty$ are positive integers, $h_{i j} \in R^{n_{1}}$ and $v_{i j} \in R^{n_{2}}$ are given vectors.

In this paper, the switch can be assumed to occur only at each sampling points of $i$ or $j$. The switching sequence can be described as

$$
\left(\left(i_{0}, j_{0}\right), \sigma\left(i_{0}, j_{0}\right)\right),\left(\left(i_{1}, j_{1}\right), \sigma\left(i_{1}, j_{1}\right)\right), \ldots,\left(\left(i_{\pi}, j_{\pi}\right), \sigma\left(i_{\pi}, j_{\pi}\right)\right), \ldots,
$$

where $\left(i_{\pi}, j_{\pi}\right)$ denotes the $\pi$-th switching instant. It should be noted that the value of $\sigma(i, j)$ only depends upon $i+j$ (see the references $[5,47])$.

Definition 1 [11]. System (1) is called a positive 2D switched model if $x^{h}(i, j) \succeq 0$ and $x^{v}(i, j) \succeq 0$ for any nonnegative boundary conditions $h_{i j} \in R_{+}^{n_{1}}$ and $v_{i j} \in R_{+}^{n_{2}}$.

The following lemma is a direct extension from positive 2D systems in [21] to $2 \mathrm{D}$ switched positive systems.

Lemma 1 [21]. System (1) is positive if and only if $A^{k} \succeq 0, A_{d}^{k} \succeq 0$ and $B^{k} \succeq 0$.

Remark 1. When $N=1$, positive 2D switched system (1) will degenerate into the following positive 2D system [26].

$$
\left[\begin{array}{l}
x^{h}(i+1, j) \\
x^{v}(i, j+1)
\end{array}\right]=A\left[\begin{array}{l}
x^{h}(i, j) \\
x^{v}(i, j)
\end{array}\right]+A_{d}\left[\begin{array}{l}
x^{h}\left(i-d_{h}(i), j\right) \\
x^{v}\left(i, j-d_{v}(j)\right)
\end{array}\right]+B u(i, j) .
$$

Definition 2 [18]. System (1) with $u(i, j)=0$ is said to be exponentially stable under the switching signal $\sigma(\cdot)$, if for a given $z \geqslant 0$, there exist positive constants $c$ and $\xi$ such that

$$
\sum_{i+j=D}\|x(i, j)\| \leqslant \xi e^{-c(D-z)} \sum_{i+j=z}\|x(i, j)\|_{C},
$$

holds for all $D \geqslant z$, where

$$
\begin{aligned}
& \sum_{i+j=z}\|x(i, j)\|_{C} \triangleq \sup _{\substack{-d_{h H} \leqslant \theta_{h} \leqslant 0 i+j=z \\
-d_{v H} \leqslant v \leqslant 0}}\left\{\left\|x\left(i-\theta_{h}, j\right)\right\|,\left\|x\left(i, j-\theta_{v}\right)\right\|,\left\|\delta^{h}\left(i-\theta_{h}, j\right)\right\|,\left\|\delta^{v}\left(i, j-\theta_{v}\right)\right\|\right\}, \\
& \delta^{h}\left(i-\theta_{h}, j\right)=x^{h}\left(i-\theta_{h}+1, j\right)-x^{h}\left(i-\theta_{h}, j\right), \\
& \delta^{v}\left(i, j-\theta_{v}\right)=x^{v}\left(i, j-\theta_{v}+1\right)-x^{v}\left(i, j-\theta_{v}\right) .
\end{aligned}
$$

Remark 2. From Definition 2, it is easy to see that, for a given $z, \sum_{i+j=z}\|x(i, j)\|_{C}$ will be bounded, and $\sum_{i+j=D}\|x(i, j)\|$ will tend to be zero exponentially as $D$ goes to infinity, which also means $\|x(i, j)\|$ will tend to be zero exponentially.

Definition 3. [47]. For any $i+j=D \geqslant z=i_{z}+j_{z}$, let $N_{\sigma}(z, D)$ denote the switching number of $\sigma(\cdot)$ on an interval $[z, D)$. If

$$
N_{\sigma}(z, D) \leqslant N_{0}+\frac{D-z}{\tau_{a}}
$$

holds for given $N_{0} \geqslant 0$ and $\tau_{a} \geqslant 0$, then the constant $\tau_{a}$ is called the average dwell time and $N_{0}$ is the chatter bound. As commonly used in the literature, we choose $N_{0}=0$ in this paper.

Remark 3. Definition 3 gives a definition of average dwell time for 2D switched discrete systems and the definition can be viewed as an extension of the proposed one in 1D (one-dimensional) switched systems. Similarly, if there exists a positive number $\tau_{a}$ such that a switching signal has the average dwell time property, the average time interval between consecutive switching is at least $\tau_{a}$.

The aim of this paper is to design a state feedback controller for system (1) such that the resulting closed-loop system is positive and exponentially stable.

\section{Main results}

\subsection{Stability analysis}

In order to address the control problem, we first focus on the problem of delay-dependent exponential stability analysis for the following positive 2D switched discrete linear systems with state delays 


$$
\left[\begin{array}{l}
x^{h}(i+1, j) \\
x^{v}(i, j+1)
\end{array}\right]=A^{\sigma(i, j)}\left[\begin{array}{l}
x^{h}(i, j) \\
x^{v}(i, j)
\end{array}\right]+A_{d}^{\sigma(i, j)}\left[\begin{array}{l}
x^{h}\left(i-d_{h}(i), j\right) \\
x^{v}\left(i, j-d_{v}(j)\right)
\end{array}\right]
$$

Theorem 1. For given positive constants $d_{h L}, d_{h H}, d_{v L}, d_{v H}$, and a scalar $0<\alpha<1$, if there exist vectors $p^{k} \in R_{+}^{n}, q^{k} \in R_{+}^{n}, \varsigma_{1}^{k} \in R_{+}^{n}$, $\varsigma_{2}^{k} \in R_{+}^{n}, \zeta^{k} \in R_{+}^{n}, k \in \underline{N}$, such that

$$
\Phi^{k}=\operatorname{diag}\left\{\Phi_{1}^{k}, \Phi_{2}^{k}, \ldots, \Phi_{n}^{k}, \Phi_{1}^{k^{\prime}}, \Phi_{2}^{k^{\prime}}, \ldots, \Phi_{n}^{k^{\prime}}, \Phi_{1}^{k^{\prime \prime}}, \Phi_{2}^{k^{\prime \prime}}, \ldots, \Phi_{n}^{k^{\prime \prime}}, \Phi_{1}^{k^{\prime \prime \prime}}, \Phi_{2}^{k^{\prime \prime \prime}}, \ldots, \Phi_{n}^{k^{\prime \prime \prime}}\right\}<0, \quad \forall k \in \underline{N}
$$

where

$$
\begin{aligned}
& \Phi_{l}^{k}= \begin{cases}\left(a_{l}^{k T}-\alpha E_{l}\right) p^{k}+\left(\alpha^{-1} a_{l}^{k T}+\left(d_{h H}-d_{h L}\right) E_{l}\right) q^{k}+E_{l} \zeta^{k}+d_{h H}^{2} E_{l} \varsigma_{1}^{k}+\left(d_{h H}^{2}\left(a_{l}^{k T}-E_{l}\right)-d_{h H} \alpha^{d_{h H}} E_{l}\right) \varsigma_{2}^{k}, & 1 \leqslant l \leqslant n_{1}, \\
\left(a_{l}^{k T}-\alpha E_{l}\right) p^{k}+\left(\alpha^{-1} a_{l}^{k T}+\left(d_{v H}-d_{v L}\right) E_{l}\right) q^{k}+E_{l} \zeta^{k}+d_{v H}^{2} E_{l} \varsigma_{1}^{k}+\left(d_{v H}^{2}\left(a_{l}^{k T}-E_{l}\right)-d_{v H} \alpha^{d_{\nu H}} E_{l}\right) \varsigma_{2}^{k}, & n_{1}+1 \leqslant l \leqslant n,\end{cases} \\
& \Phi_{l}^{k^{\prime}}= \begin{cases}a_{d l}^{k T} p^{k}+\left(\alpha^{-1} a_{d l}^{k T}-\alpha^{d_{h H}} E_{l}\right) q^{k}+d_{h H}^{2} a_{d l}^{k T} \varsigma_{2}^{k}, & 1 \leqslant l \leqslant n_{1}, \\
a_{d l}^{k T} p^{k}+\left(\alpha^{-1} a_{d l}^{k T}-\alpha^{d_{v H}} E_{l}\right) q^{k}+d_{v H}^{2} a_{d l}^{k T} s_{2}^{k}, & n_{1}+1 \leqslant l \leqslant n,\end{cases} \\
& \Phi_{l}^{k^{\prime \prime}}= \begin{cases}-\alpha^{d_{h H}} E_{l} \zeta^{k}+\alpha^{d_{h H}} d_{h H} E_{l}\left(\varsigma_{2}^{k}-\varsigma_{1}^{k}\right), & 1 \leqslant l \leqslant n_{1}, \\
-\alpha^{d_{\nu H}} E_{l} \zeta^{k}+\alpha^{d_{\nu H}} d_{v H} E_{l}\left(\varsigma_{2}^{k}-\varsigma_{1}^{k}\right), & n_{1}+1 \leqslant l \leqslant n,\end{cases} \\
& \Phi_{l}^{k^{\prime \prime \prime}}= \begin{cases}-\alpha^{d_{h H}} d_{h H} E_{l} \zeta_{1}^{k}, & 1 \leqslant l \leqslant n_{1}, \\
-\alpha^{d_{v H}} d_{v H} E_{l} \zeta_{1}^{k}, & n_{1}+1 \leqslant l \leqslant n,\end{cases}
\end{aligned}
$$

with $l \in \underline{n}=\{1,2, \ldots, n\}, E_{l}=[\overbrace{0, \ldots, 0}^{l-1}, 1, \overbrace{0, \ldots, 0}^{n-l}]$, and $a_{l}^{k}\left(a_{d l}^{k}\right)$ represents the l-th column vector of matrix $A^{k}\left(A_{d}^{k}\right)$, then system (6) is exponentially stable for any switching signals with the average dwell time satisfying

$$
\tau_{a}>\tau_{a}^{*}=\frac{\ln \mu}{-\ln \alpha},
$$

where $\mu \geqslant 1$ satisfies

$$
p^{k} \prec \mu p^{f}, q^{k} \prec \mu q^{f}, \zeta^{k} \prec \mu \zeta^{f}, \varsigma_{1}^{k} \prec \mu \varsigma_{1}^{f}, \varsigma_{2}^{k} \prec \mu \varsigma_{2}^{f}, \forall k, f \in \underline{N},
$$

Proof. Without loss of generality, we assume that the $k$-th subsystem is active. For the $k$-th subsystem, we choose the following co-positive Lyapunov-Krasovskii functional candidate

$$
V^{k}(i, j)=V^{k h}(i, j)+V^{k v}(i, j),
$$

where

$$
V^{k h}(i, j)=\sum_{g=1}^{5} V_{g}^{k h}(i, j)
$$

with

$$
\begin{aligned}
& V_{1}^{k h}(i, j)=x^{h T}(i, j) p^{k h}, \\
& V_{2}^{k h}(i, j)=\sum_{r=i-d_{h}(i)}^{i} \alpha^{i-1-r} \chi^{h T}(r, j) q^{k h}, V_{3}^{k h}(i, j)=\sum_{r=i-d_{h H}}^{i-1} \alpha^{i-1-r} \chi^{h T}(r, j) \xi^{k h}, \\
& V_{4}^{k h}(i, j)=\sum_{s=-d_{h H}+1}^{-d_{h L}} \sum_{r=i+s}^{i-1} \alpha^{i-1-r} \chi^{h T}(r, j) q^{k h}, V_{5}^{k h}(i, j)=d_{h H} \sum_{s=-d_{h H}}^{-1} \sum_{r=i+s}^{i-1} \alpha^{i-1-r} \eta^{h T}(r, j) \xi^{k h},
\end{aligned}
$$

and 


$$
V^{k v}(i, j)=\sum_{g=1}^{5} V_{g}^{k v}(i, j),
$$

with

$$
\begin{aligned}
& V_{1}^{k v}(i, j)=x^{v T}(i, j) p^{k v} \\
& V_{2}^{k v}(i, j)=\sum_{t=j-d_{\nu}(j)}^{j} \alpha^{j-1-t} x^{v T}(i, t) q^{k v}, V_{3}^{k v}(i, j)=\sum_{t=j-d_{\nu \mathrm{H}}}^{j-1} \alpha^{j-1-t} \chi^{v \mathrm{~T}}(i, t) \xi^{k v}, \\
& V_{4}^{k v}(i, j)=\sum_{s=-d_{\nu H}+1}^{-d_{v L}} \sum_{t=j+s}^{j-1} \alpha^{j-1-t} \chi^{v T}(i, t) q^{k v}, V_{5}^{k v}(i, j)=d_{v H} \sum_{s=-d_{\nu H} t=j+s}^{-1} \sum^{j-1} \alpha^{j-1-t} \eta^{v T}(i, t) \varsigma^{k v},
\end{aligned}
$$

and

$$
\begin{aligned}
& \eta^{h}(r, j)=\left[\begin{array}{ll}
x^{h T}(r, j) & \delta^{h T}(r, j)
\end{array}\right]^{T}, \eta^{v}(i, t)=\left[\begin{array}{ll}
x^{v T}(i, t) & \delta^{v T}(i, t)
\end{array}\right]^{T}, \\
& \delta^{h}(r, j)=x^{h}(r+1, j)-x^{h}(r, j), \delta^{v}(i, t)=x^{v}(i, t+1)-x^{v}(i, t),
\end{aligned}
$$

with $p^{k h} \in R_{+}^{n_{1}}, q^{k h} \in R_{+}^{n_{1}}, \zeta^{k h} \in R_{+}^{n_{1}}, \varsigma_{1}^{k h} \in R_{+}^{n_{1}}, \varsigma_{2}^{k h} \in R_{+}^{n_{1}}, \varsigma^{k h}=\left[\begin{array}{cc}\varsigma_{1}^{k h T} & \varsigma_{2}^{k k T}\end{array}\right]^{T} \in R_{+}^{2 n_{1}}, \zeta^{k v} \in R_{+}^{n_{2}}, \varsigma_{1}^{v} \in R_{+}^{n_{2}}, \varsigma_{2}^{k v} \in R_{+}^{n_{2}}, \quad p^{k v} \in R_{+}^{n_{2}}$, $q^{k v} \in R_{+}^{n_{2}}$ and $\varsigma^{k v}=\left[\begin{array}{ll}\varsigma_{1}^{k v T} & \varsigma_{2}^{k v T}\end{array}\right]^{T} \in R_{+}^{2 n_{2}}$ are real vectors to be determined.

Then we have

$$
V^{k h}(i+1, j)-\alpha V^{k h}(i, j)+V^{k v}(i, j+1)-\alpha V^{k v}(i, j)=\sum_{g=1}^{5}\left[V_{g}^{k h}(i+1, j)-\alpha V_{g}^{k h}(i, j)\right]+\sum_{g=1}^{5}\left[V_{g}^{k v}(i, j+1)-\alpha V_{g}^{k v}(i, j)\right] .
$$

Along the trajectory of system (6), one can obtain

$$
\begin{aligned}
& V_{1}^{k h}(i+1, j)-\alpha V_{1}^{k h}(i, j)=x^{h T}(i+1, j) p^{k h}-\alpha x^{h T}(i, j) p^{k h}, \\
& V_{2}^{k h}(i+1, j)-\alpha V_{2}^{k h}(i, j)=\sum_{r=i+1-d_{h}(i+1)}^{i+1} \alpha^{i-r} \chi^{h T}(r, j) q^{k h}-\alpha \sum_{r=i-d_{h}(i)}^{i} \alpha^{i-1-r} \chi^{h T}(r, j) q^{k h} \\
& =\alpha^{-1} x^{h T}(i+1, j) q^{k h}-\alpha^{d_{h}(i)} x^{h T}\left(i-d_{h}(i), j\right) q^{k h}+\sum_{r=i+1-d_{h}(i+1)}^{i} \alpha^{i-r} x^{h T}(r, j) q^{k h} \\
& -\alpha \sum_{r=i+1-d_{h}(i)}^{i} \alpha^{i-1-r} \chi^{h T}(r, j) q^{k h} \\
& \leqslant \alpha^{-1} x^{h T}(i+1, j) q^{k h}-\alpha^{d_{h}(i)} x^{h T}\left(i-d_{h}(i), j\right) q^{k h}+\sum_{r=i+1-d_{h H}}^{i} \alpha^{i-r} x^{h T}(r, j) q^{k h} \\
& -\sum_{r=i+1-d_{h l}}^{i} \alpha^{i-r} x^{h T}(r, j) q^{k h} \\
& \leqslant \alpha^{-1} \chi^{h T}(i+1, j) q^{k h}-\alpha^{d_{h H}} \chi^{h T}\left(i-d_{h}(i), j\right) q^{k h}+\sum_{r=i+1-d_{h H}}^{i-d_{h l}} \alpha^{i-r} \chi^{h T}(r, j) q^{k h}, \\
& V_{3}^{k h}(i+1, j)-\alpha V_{3}^{k h}(i, j)=\sum_{r=i+1-d_{h H}}^{i} \alpha^{i-r} x^{h T}(r, j) \xi^{k h}-\alpha \sum_{r=i-d_{h H}}^{i-1} \alpha^{i-1-r} x^{h T}(r, j) \xi^{k h}=x^{h T}(i, j) \zeta^{k h}-\alpha^{d_{h H}} x^{h T}\left(i-d_{h H}, j\right) \zeta^{k h}, \\
& V_{4}^{k h}(i+1, j)-\alpha V_{4}^{k h}(i, j)=\sum_{s=-d_{h H}+1}^{-d_{h L}} \sum_{r=i+1+s}^{i} \alpha^{i-r} \chi^{h T}(r, j) q^{k h}-\alpha \sum_{s=-d_{h H}+1}^{-d_{h L}} \sum_{r=i+s}^{i-1} \alpha^{i-1-r} \chi^{h T}(r, j) q^{k h} \\
& =\sum_{s=-d_{h H}+1}^{-d_{h L}}\left[x^{h T}(i, j) q^{k h}-\alpha^{-s} x^{h T}(i+s, j) q^{k h}\right]=\left(d_{h H}-d_{h L}\right) x^{h T}(i, j) q^{k h}-\sum_{r=i-d_{h H}+1}^{i-d_{h L}} \alpha^{i-r} x^{h T}(r, j) q^{k h},
\end{aligned}
$$

and 


$$
\begin{aligned}
V_{5}^{k h}(i+1, j)-\alpha V_{5}^{k h}(i, j) & =d_{h H} \sum_{s=-d_{h H}}^{-1} \sum_{r=i+1+s}^{i} \alpha^{i-r} \eta^{h T}(r, j) \varsigma^{k h}-\alpha d_{h H} \sum_{s=-d_{h H}}^{-1} \sum_{r=i+s}^{i-1} \alpha^{i-1-r} \eta^{h T}(r, j) \varsigma^{k h} \\
& =d_{h H} \sum_{s=-d_{h H}}^{-1}\left(\eta^{h T}(i, j) \varsigma^{k h}-\alpha^{-s} \eta^{h T}(i+s, j) \varsigma^{k h}\right) \leqslant d_{h H}^{2} \eta^{h T}(i, j) \varsigma^{k h}-d_{h H} \alpha^{d_{h H}} \sum_{r=i-d_{h H}}^{i-1} \eta^{h T}(r, j) \varsigma^{k h} \\
& =d_{h H}^{2}\left[x^{h T}(i, j) x^{h T}(i+1, j)-x^{h T}(i, j)\right]\left[\begin{array}{c}
\varsigma_{1}^{k h} \\
\varsigma_{2}^{k h}
\end{array}\right]-d_{h H} \alpha^{d_{h H}}\left[\sum_{r=i-d_{h H}}^{i-1} x^{h T}(r, j) x^{h T}(i, j)-x^{h T}\left(i-d_{h H}, j\right)\right]\left[\begin{array}{c}
\varsigma_{1}^{k h} \\
\varsigma_{2}^{k h}
\end{array}\right] .
\end{aligned}
$$

Similarly, we can get the following formulations in vertical direction.

$$
\begin{aligned}
& V_{1}^{k v}(i, j+1)-\alpha V_{1}^{k v}(i, j)=x^{v T}(i, j+1) p^{k v}-\alpha x^{v T}(i, j) p^{k v}, \\
& V_{2}^{k v}(i, j+1)-\alpha V_{2}^{k v}(i, j) \leqslant \alpha^{-1} x^{v T}(i, j+1) q^{k v}-\alpha^{d_{\nu H}} \chi^{v T}\left(i, j-d_{v}(j)\right) q^{k v}+\sum_{t=j+1-d_{v H}}^{j-d_{\nu L}} \alpha^{j-t} x^{v T}(i, t) q^{k v}, \\
& V_{3}^{k v}(i, j+1)-\alpha V_{3}^{k v}(i, j)=x^{v T}(i, j) \xi^{k v}-\alpha^{d_{v H}} x^{v T}\left(i, j-d_{v H}\right) \zeta^{k v}, \\
& V_{4}^{k v}(i, j+1)-\alpha V_{4}^{k v}(i, j)=\left(d_{v H}-d_{v L}\right) x^{v \mathrm{~T}}(i, j) q^{k v}-\sum_{t=j-d_{v H}+1}^{j-d_{v L}} \alpha^{j-t} x^{v \mathrm{~T}}(i, t) q^{k v}, \\
& V_{5}^{k v}(i, j+1)-\alpha V_{5}^{k v}(i, j) \leqslant d_{v H}^{2}\left[x^{v T}(i, j) \quad x^{v T}(i, j+1)-x^{v T}(i, j)\right]\left[\begin{array}{c}
\varsigma_{1}^{k v} \\
\varsigma_{2}^{k v}
\end{array}\right] \\
& -d_{v H} \alpha^{d_{\nu H}}\left[\sum_{t=j-d_{v H}}^{j-1} x^{v T}(i, t) \quad x^{v T}(i, j)-x^{v T}\left(i, j-d_{v H}\right)\right]\left[\begin{array}{c}
\varsigma_{1}^{k \nu} \\
\varsigma_{2}^{k v}
\end{array}\right] .
\end{aligned}
$$

Substitute the above formulations (10)-(19) into (8), and take

$$
\begin{aligned}
& p^{k}=\left[\begin{array}{l}
p^{k h} \\
p^{k v}
\end{array}\right], q^{k}=\left[\begin{array}{c}
q^{k h} \\
q^{k v}
\end{array}\right], \zeta^{k}=\left[\begin{array}{c}
\zeta^{k h} \\
\zeta^{k v}
\end{array}\right], \varsigma_{1}^{k}=\left[\begin{array}{c}
\varsigma_{1}^{k h} \\
\varsigma_{1}^{k v}
\end{array}\right], \varsigma_{2}^{k}=\left[\begin{array}{c}
\varsigma_{2}^{k h} \\
\varsigma_{2}^{k v}
\end{array}\right], \\
& D_{H}=\left[\begin{array}{cc}
d_{h H} I_{n_{1}} & 0 \\
0 & d_{v H} I_{n_{2}}
\end{array}\right], D_{L}=\left[\begin{array}{cc}
d_{h L} I_{n_{1}} & 0 \\
0 & d_{v L} I_{n_{2}}
\end{array}\right], \Omega=\left[\begin{array}{cc}
\alpha^{d_{h H} I_{n_{1}}} & 0 \\
0 & \alpha^{d} I I_{n_{2}}
\end{array}\right] \text {, } \\
& x(i, j)=\left[\begin{array}{ll}
x^{h T}(i, j) & x^{v T}(i, j)
\end{array}\right]^{T}, \\
& x_{d}(i, j)=\left[\begin{array}{ll}
x^{h T}\left(i-d_{h}(i), j\right) & x^{v T}\left(i, j-d_{v}(j)\right)
\end{array}\right]^{T}, \\
& x_{H}(i, j)=\left[x^{h T}\left(i-d_{h H}, j\right) \quad x^{v T}\left(i, j-d_{v H}\right)\right]^{T}, \\
& x_{s}(i, j)=\left[\sum_{r=i-d_{h H}+1}^{i-1} x^{h T}(r, j) \sum_{t=j-d_{\nu H+1}}^{j-1} x^{v T}(i, t)\right]^{T} .
\end{aligned}
$$

Then we have

$$
\begin{aligned}
V^{k h}(i+1, j)-\alpha V^{k h}(i, j)+V^{k v}(i, j+1)-\alpha V^{k v}(i, j)= & x^{T}(i, j)\left\{\left(A^{k T}-\alpha I_{n}\right) p^{k}+\left(\alpha^{-1} A^{k T}+\left(D_{H}-D_{L}\right)\right) q^{k}\right. \\
& \left.+\zeta^{k}+D_{H}^{2} \varsigma_{1}^{k}+\left(D_{H}^{2} A^{k T}-D_{H}^{2}-\Omega D_{H}\right) \varsigma_{2}^{k}\right\}+x_{d}^{T}(i, j)\left\{A_{d}^{k T} p^{k}+\left(\alpha^{-1} A_{d}^{k T}-\Omega\right) q^{k}+D_{H}^{2} A_{d}^{k T} \varsigma_{2}^{k}\right\} \\
& +x_{H}^{T}(i, j)\left\{-\Omega \zeta^{k}+\Omega D_{H} \varsigma_{2}^{k}-\Omega D_{H} \varsigma_{1}^{k}\right\}+x_{s}^{T}(i, j)\left\{-\Omega D_{H} \varsigma_{1}^{k}\right\} .
\end{aligned}
$$

If condition (7a) holds, one obtains

$$
\begin{aligned}
& \left(A^{k T}-\alpha I_{n}\right) p^{k}+\left(\alpha^{-1} A^{k T}+\left(D_{H}-D_{L}\right)\right) q^{k}+\zeta^{k}+D_{H}^{2} \varsigma_{1}^{k}+\left(D_{H}^{2} A^{k T}-D_{H}^{2}-\Omega D_{H}\right) \varsigma_{2}^{k} \prec 0, \\
& A_{d}^{k T} p^{k}+\left(\alpha^{-1} A_{d}^{k T}-\Omega\right) q^{k}+D_{H}^{2} A_{d}^{k T} \varsigma_{2}^{k} \prec 0, \\
& -\Omega \zeta^{k}+\Omega D_{H} \varsigma_{2}^{k}-\Omega D_{H} \varsigma_{1}^{k} \prec 0, \\
& -\Omega D_{H} \varsigma_{1}^{k} \prec 0 .
\end{aligned}
$$


Inequalities (21)-(24) imply that

$$
V^{k h}(i+1, j)+V^{k v}(i, j+1)<\alpha V^{k h}(i, j)+\alpha V^{k v}(i, j) .
$$

Summing up both sides of (25) from $D$ to 0 with respect to $i$ and 0 to $D$ with respect to $j$, for any nonnegative integer $D>\max$ $\left(z_{1}, z_{2}\right)$, one gets

$$
\begin{aligned}
& V^{k h}(1, D)+V^{k v}(0, D+1)+V^{k h}(2, D-1)+V^{k v}(1, D)+\cdots+V^{k h}(D+1,0)+V^{k v}(D, 1) \\
& =\sum_{i+j=D+1} V^{k h}(i, j)+\sum_{i+j=D+1} V^{k v}(i, j)=\sum_{i+j=D+1} V^{k}(i, j)<\alpha\left\{V^{k h}(0, D)+V^{k v}(0, D)+V^{k h}(1, D-1)\right. \\
& \left.\quad+V^{k v}(1, D-1)+\cdots+V^{k h}(D, 0)+V^{k v}(D, 0)\right\}=\alpha \sum_{i+j=D} V^{k}(i, j) .
\end{aligned}
$$

Now let $v=N_{\sigma}(z, D)$ denote the switching number of $\sigma(\cdot)$ on an interval $[z, D)$, and let $\left(i_{\kappa-v+1}, j_{\kappa-v+1}\right),\left(i_{\kappa-v+2}, j_{\kappa-v+2}\right)$, $\ldots\left(i_{\kappa-1}, j_{\kappa-1}\right),\left(i_{\kappa}, j_{\kappa}\right)$ denote the switching points of $\sigma(\cdot)$ over the interval $[z, D)$. Denoting $m_{p}=i_{p}+j_{p}, p=\kappa-v+1$, $\kappa-v+2, \ldots, \kappa$, thus, for $D \in\left[m_{\kappa}, m_{\kappa+1}\right)$, it holds from (26) that

$$
\sum_{i+j=D} V^{\sigma\left(i_{\kappa} j_{\kappa}\right)}(i, j)<\alpha^{D-m_{\kappa}} \sum_{i+j=m_{\kappa}} V^{\sigma\left(i_{\kappa} j_{\kappa}\right)}(i, j) .
$$

Using (7c) and (8), at switching instant $m_{\kappa}=i+j$, we have

$$
\sum_{i+j=m_{\kappa}} V^{\sigma\left(i_{\kappa} \cdot j_{\kappa}\right)}(i, j) \leqslant \mu \sum_{i+j=m_{\kappa}} V^{\sigma\left(i_{\kappa-1} j_{\kappa-1}\right)}(i, j) .
$$

In addition, according to Definition 3, it follows that

$$
v=N_{\sigma}(z, D) \leqslant N_{0}+\frac{D-z}{\tau_{a}} .
$$

Therefore, the following inequality can be easily obtained by repeating the inequalities (27), (28) and using (29)

$$
\begin{aligned}
\sum_{i+j=D} V^{\sigma\left(i_{\kappa} j_{\kappa}\right)}(i, j) & <\alpha^{D-m_{\kappa}} \sum_{i+j=m_{\kappa}} V^{\sigma\left(i_{\kappa} j_{\kappa}\right)}(i, j) \leqslant \mu \alpha^{D-m_{\kappa}} \sum_{i+j=m_{\kappa}^{-}} V^{\sigma\left(i_{\kappa-1} j_{\kappa-1}\right)}(i, j)<\mu \alpha^{D-m_{\kappa}} \sum_{i+j=m_{\kappa-1}} V^{\sigma\left(i_{\kappa-1} j_{\kappa-1}\right)}(i, j) \alpha^{m_{\kappa}-m_{\kappa-1}} \\
& =\mu \alpha^{D-m_{\kappa-1}} \sum_{i+j=m_{\kappa-1}} V^{\sigma\left(i_{\kappa-1} j_{\kappa-1}\right)}(i, j) \leqslant \cdots<\mu^{v-1} \alpha^{D-m_{\kappa-v+1}} \sum_{i+j=m_{\kappa-v+1}} V^{\sigma\left(i_{\kappa-v+1} j_{\kappa-v+1}\right)}(i, j) \\
& \leqslant \mu^{v} \alpha^{D-m_{\kappa-v+1}} \sum_{i+j=m_{k-v+1}^{-}} V^{\sigma\left(i_{\kappa-v} j_{\kappa-v}\right)}(i, j)=\mu^{v} \alpha^{D-m_{k-v+1}} \sum_{i+j=z} V^{\sigma\left(i_{\kappa-v} j_{\kappa-v}\right)}(i, j) \alpha^{m_{\kappa-v+1}-z} \\
& \leqslant \mu^{v} \alpha^{D-z} \sum_{i+j=z} V^{\sigma\left(i_{\kappa-v} j_{\kappa-v}\right)}(i, j) .
\end{aligned}
$$

Inequality (30) can be rewritten as follows:

$$
\sum_{i+j=D} V^{\sigma\left(i_{\kappa}, j_{\kappa}\right)}(i, j) \leqslant e^{\left.\frac{(\ln \mu}{\tau_{a}}+\ln \alpha\right)(D-z)} \sum_{i+j=z} V^{\sigma\left(i_{K-v}, j_{\kappa-v}\right)}(i, j) .
$$

Moreover, considering the definition of $V^{\sigma(i, j)}(i, j)$ in (8), we can find two positive scalars $\rho_{1}$ and $\rho_{2}$ such that (32) holds.

$$
\rho_{1}\|x(i, j)\| \leqslant V^{\sigma(i, j)}(i, j) \leqslant \rho_{2}\|x(i, j)\|_{C},
$$

where

$$
\begin{aligned}
& \rho_{1}=\min _{(l, k) \in \underline{n} \times \underline{N} \underline{N}}\left(p_{l}^{k}\right), \\
& \rho_{2}=\max _{(l, k) \in \underline{n} \times \underline{N}} p_{l}^{k}+\left[\frac{d_{H}}{2}\left(d_{H}+d_{L}-1\right)\left(d_{H}-d_{L}\right)+d_{H}\right] \max _{(l, k) \in \underline{n} \times \underline{N}} q_{l}^{k}+d_{H} \max _{(l, k) \in \underline{n} \times \underline{N} \underline{N}} \zeta_{l}^{k}+d_{H}^{2} \max _{(l, k) \in \underline{n} \times \underline{N}} s_{1 l}^{k}+d_{H}\left(d_{H}+1\right) \max _{(l, k) \in \underline{n} \times \underline{N} \underline{N} \zeta_{2 l}^{k}}
\end{aligned}
$$

with

$$
\begin{aligned}
d_{H} & =\max \left(d_{h H}, d_{v H}\right), d_{L}=\min \left(d_{h L}, d_{v L}\right), p^{k}=\left[p_{1}^{k}, p_{2}^{k}, \ldots, p_{n}^{k}\right]^{T}, \\
q^{k} & =\left[q_{1}^{k}, q_{2}^{k}, \ldots, q_{n}^{k}\right]^{T}, \zeta^{k}=\left[\zeta_{1}^{k}, \zeta_{2}^{k}, \ldots, \zeta_{n}^{k}\right]^{T}, \varsigma_{1}^{k}=\left[\zeta_{11}^{k}, \varsigma_{12}^{k}, \ldots, \varsigma_{1 n}^{k}\right]^{T}, \\
\varsigma_{2}^{k} & =\left[\varsigma_{21}^{k}, \varsigma_{22}^{k}, \ldots, \varsigma_{2 n}^{k}\right]^{T} .
\end{aligned}
$$

Combining (31) and (32), one can obtain

$$
\sum_{i+j=D}\|x(i, j)\| \leqslant \frac{\rho_{2}}{\rho_{1}} e^{\left(\frac{\ln \mu}{\tau_{a}}+\ln \alpha\right)(D-z)} \sum_{i+j=z}\|x(i, j)\|_{C} .
$$


By Definition 2, we know that the positive 2D switched discrete system is exponentially stable if $\tau_{a}>\tau_{a}^{*}=\frac{\ln \mu}{-\ln \alpha}$. This completes the proof.

Remark 4. Note that when $\mu=1$ in (7b), we have $\tau_{a}^{*}=0$, which means that the switching signal can be arbitrary.

Remark 5. It should be noted that a co-positive Lyapunov functional is constructed for the stability analysis in the derivation of Theorem 1. The motivation for using this type of Lyapunov functional is that the state of system (1) is nonnegative and hence such a linear Lyapunov functional serves as a valid candidate. Compared with the existing stability result in [11], the one presented here is in the form of LMIs which can be conveniently verified. However, there exists the conservatism induced by Lyapunov functional (8) to some extent. The result can be improved by resorting to the delay-partition method for which a modified Lyapunov functional could be chosen.

\subsection{Controller synthesis}

This subsection studies the stabilization problem of positive 2D switched discrete Roesser model (1) for which the control law to be designed has the following state-feedback form

$$
u(i, j)=K^{\sigma(i, j)}\left[\begin{array}{l}
x^{h}(i, j) \\
x^{v}(i, j)
\end{array}\right]
$$

This control law will be designed to ensure the positivity and the exponential stability of the resulting closed-loop system:

$$
\left[\begin{array}{l}
x^{h}(i+1, j) \\
x^{v}(i, j+1)
\end{array}\right]=\left(A^{\sigma(i, j)}+B^{\sigma(i, j)} K^{\sigma(i, j)}\right)\left[\begin{array}{l}
x^{h}(i, j) \\
x^{v}(i, j)
\end{array}\right]+A_{d}^{\sigma(i, j)}\left[\begin{array}{l}
x^{h}\left(i-d_{h}(i), j\right) \\
x^{v}\left(i, j-d_{v}(j)\right)
\end{array}\right] .
$$

The following lemma will be useful in the subsequent development.

Lemma 2. Given the open-loop positive system (1) and the controller given by (34), the closed-loop system (35) is positive if and only if the following conditions hold for all $k \in \underline{N}$

$$
\begin{aligned}
& A^{k}+B^{k} K^{k} \succeq 0, \\
& A_{d}^{k} \succeq 0 .
\end{aligned}
$$

Proof. The result can be obtained by applying Theorem 8 in [26] to system (35).

Theorem 2. For given positive constants $d_{h L}, d_{h H}, d_{v L}, d_{v H}$ and a scalar $0<\alpha<1$, if there exist vectors $p^{k} \in R_{+}^{n}, q^{k} \in R_{+}^{n}, \varsigma_{1}^{k} \in R_{+}^{n}, \varsigma_{2}^{k} \in R_{+}^{n}, \zeta^{k} \in R_{+}^{n}, \psi^{k} \in R_{+}^{n}$, such that

$$
\widetilde{\boldsymbol{\Phi}}^{k}=\operatorname{diag}\left\{\widetilde{\boldsymbol{\Phi}}_{1}^{k}, \widetilde{\boldsymbol{\Phi}}_{2}^{k}, \ldots, \widetilde{\boldsymbol{\Phi}}_{n}^{k}, \widetilde{\boldsymbol{\Phi}}_{1}^{k^{\prime}}, \widetilde{\boldsymbol{\Phi}}_{2}^{k^{\prime}}, \ldots, \widetilde{\boldsymbol{\Phi}}_{n}^{k^{\prime}}, \widetilde{\boldsymbol{\Phi}}_{1}^{k^{\prime \prime}}, \widetilde{\boldsymbol{\Phi}}_{2}^{k^{\prime \prime}}, \ldots, \widetilde{\boldsymbol{\Phi}}_{n}^{k^{\prime \prime}}, \widetilde{\boldsymbol{\Phi}}_{1}^{k^{\prime \prime \prime}}, \widetilde{\boldsymbol{\Phi}}_{2}^{k^{\prime \prime \prime}}, \ldots, \widetilde{\boldsymbol{\Phi}}_{n}^{k^{\prime \prime \prime}}\right\}<0, \quad \forall k \in N
$$

where

$$
\begin{aligned}
& \widetilde{\boldsymbol{\Phi}}_{l}^{k}=\left\{\begin{array}{l}
\left(a_{l}^{k T}-\alpha E_{l}\right) p^{k}+\left(\alpha^{-1} a_{l}^{k T}+\left(d_{h H}-d_{h L}\right) E_{l}\right) q^{k}+E_{l} \zeta^{k}+d_{h H}^{2} E_{l} \varsigma_{1}^{k}+\left(d_{h H}^{2}\left(a_{l}^{k T}-E_{l}\right)-d_{h H} \alpha^{d_{h H}} E_{l}\right) \varsigma_{2}^{k}+E_{l} \psi^{k}, \quad 1 \leqslant l \leqslant n_{1}, \\
\left(a_{l}^{k T}-\alpha E_{l}\right) p^{k}+\left(\alpha^{-1} a_{l}^{k T}+\left(d_{v H}-d_{v L}\right) E_{l}\right) q^{k}+E_{l} \zeta^{k}+d_{v H}^{2} E_{l} \varsigma_{1}^{k}+\left(d_{v H}^{2}\left(a_{l}^{k T}-E_{l}\right)-d_{v H} \alpha^{d_{\nu H}} E_{l}\right) \varsigma_{2}^{k}+E_{l} \psi^{k}, \quad n_{1}+1 \leqslant l \leqslant n,
\end{array}\right. \\
& \widetilde{\boldsymbol{\Phi}}_{l}^{k^{\prime}}= \begin{cases}a_{d l}^{k T} p^{k}+\left(\alpha^{-1} a_{d l}^{k T}-\alpha^{d_{h H}} E_{l}\right) q^{k}+d_{h H}^{2} a_{d l}^{k T} \varsigma_{2}^{k}, & 1 \leqslant l \leqslant n_{1}, \\
a_{d l}^{k T} p^{k}+\left(\alpha^{-1} a_{d l}^{k T}-\alpha^{d_{\nu H}} E_{l}\right) q^{k}+d_{v H}^{2} a_{d l}^{k T} \varsigma_{2}^{k}, & n_{1}+1 \leqslant l \leqslant n\end{cases} \\
& \widetilde{\boldsymbol{\Phi}}_{l}^{k^{\prime \prime}}= \begin{cases}-\alpha^{d_{h H}} E_{l} \zeta^{k}+\alpha^{d_{h H}} d_{h H} E_{l}\left(\varsigma_{2}^{k}-\varsigma_{1}^{k}\right), & 1 \leqslant l \leqslant n_{1}, \\
-\alpha^{d_{v H}} E_{l} \zeta^{k}+\alpha^{d_{v H}} d_{v H} E_{l}\left(\varsigma_{2}^{k}-\varsigma_{1}^{k}\right), & n_{1}+1 \leqslant l \leqslant n,\end{cases} \\
& \widetilde{\boldsymbol{\Phi}}_{l}^{k^{\prime \prime \prime}}= \begin{cases}-\alpha^{d_{h H}} d_{h H} E_{l} \varsigma_{1}^{k}, & 1 \leqslant l \leqslant n_{1}, \\
-\alpha^{d_{\nu H}} d_{v H} E_{l} \varsigma_{1}^{k}, & n_{1}+1 \leqslant l \leqslant n,\end{cases} \\
& \psi^{k}=K^{k T} B^{k T}\left(p^{k}+\alpha^{-1} q^{k}+D_{H}^{2} \varsigma_{2}^{k}\right),
\end{aligned}
$$


with $D_{H}=\operatorname{diag}\left\{d_{h H} I_{n_{1}}, d_{v H} I_{n_{2}}\right\}, l \in n=\{1,2, \ldots, n\}, E_{l}=\overbrace{0, \ldots, 0}^{l-1}, 1, \overbrace{0, \ldots, 0}^{n-l}]$, and $a_{l}^{k}\left(a_{d l}^{k}\right)$ represents the l-th column vector of matrix $A^{k}\left(A_{d}^{k}\right)$, then the closed-loop system (35) is positive and exponentially stable for any switching signals with the average dwell time satisfying (7b) and (7c). Under the above conditions, the desired controller gain matrices can be computed by $\psi^{k}=K^{k T} B^{k T}\left(p^{k}+\alpha^{-1} q^{k}+D_{H}^{2} \varsigma_{2}^{k}\right)$.

Proof. From Theorem 1 and Lemma 2, the closed-loop system (35) is positive and exponentially stable if conditions (7b), (7c) and the following conditions hold.

$$
\begin{aligned}
& \left(A^{k T}-\alpha I_{n}\right) p^{k}+\left(\alpha^{-1} A^{k T}+\left(D_{H}-D_{L}\right)\right) q^{k}+\zeta^{k}+D_{H}^{2} \varsigma_{1}^{k}+\left(A^{k T} D_{H}^{2}-D_{H}^{2}-\Omega D_{H}\right) \varsigma_{2}^{k}+\psi^{k} \prec 0, \\
& A_{d}^{k T} p^{k}+\left(\alpha^{-1} A_{d}^{k T}-\Omega\right) q^{k}+A_{d}^{k T} D_{H}^{2} \varsigma_{2}^{k} \prec 0, \\
& -\Omega \zeta^{k}+\Omega D_{H} \varsigma_{2}^{k}-\Omega D_{H} \varsigma_{1}^{k} \prec 0, \\
& -\Omega D_{H} \varsigma_{1}^{k} \prec 0,
\end{aligned}
$$

where $\psi^{k}=K^{k T} B^{k T}\left(p^{k}+\alpha^{-1} q^{k}+D_{H}^{2} \zeta_{2}^{k}\right) \succ 0$, and $K^{k} \succ 0, k \in \underline{N}$.

Then the conditions in (36) can be easily obtained from (37)-(40). This completes the proof.

The procedure for constructing the desired controller is given below.

\section{Algorithm 1.}

Step 1. Solve the LMIs in (36) to obtain $p^{k}, q^{k}, \varsigma_{1}^{k}, \varsigma_{2}^{k}, \zeta^{k}$ and $\psi^{k}, k \in \underline{N}$.

Step 2. By $\psi^{k}=K^{k T} B^{k T}\left(p^{k}+\alpha^{-1} q^{k}+D_{H}^{2} \varsigma_{2}^{k}\right)$, compute $K^{k}, k \in \underline{N}$.

Step 3. Compute $\mu$ and $\tau_{a}^{*}$ by (7c) and (7b).

Step 4. The desired positive state feedback controller can be given as (34) with the obtained $K^{k}, k \in \underline{N}$.

Remark 6. The condition (36) in Theorem 2 is given in terms of LMIs which are computationally tractable by using the LMI toolbox and it is different from the results presented in [11], where the algebraic method is utilized for the case without state delays. Also, this paper takes time delay into consideration firstly, which is universal in the application.

Remark 7. We would like to point out that the algorithm we proposed may bring some computational complexities. It can be seen that we need to solve $N$ matrix inequalities to obtain $6 N$ variables in (36).

Remark 8. Many practical complicated systems can be modeled by the addressed system in this paper, among which a typical example is the thermal process under the standard measurement of absolute temperature. The features of positivity for state, switching for the control law, and complexity for multi-dimensional systems constitute a challenging problem in the control field. The co-positive type Lyapunov functional method and typical average dwell time method are merged together to solve this significant problem in this paper. The proposed LMI approach is easy to be extended to more complex applications. The feasibility of the proposed method will be illustrated by the example given in the next section.

\section{Example}

In this section, we present an example to illustrate the effectiveness of the proposed approach. Consider the thermal processes in chemical reactors with two modes, which can be expressed in the following partial differential equation with time delays. We assume that one can switch from a mode to another mode arbitrarily.

$$
\frac{\partial T(x, t)}{\partial x}=-\frac{\partial T(x, t)}{\partial t}-a_{0}^{\sigma(x, t)} T(x, t)-a_{1}^{\sigma(x, t)} T(x, t-\tau)+b^{\sigma(x, t)} u(x, t),
$$

where $T(x, t)$ is the temperature at $x \in\left[0, x_{f}\right]$ (space) and $t \in[0, \infty)$ (time), $u(x, t)$ is the input function, $\tau$ is the time delay, and $a_{0}^{\sigma(x, t)}, a_{1}^{\sigma}, b^{\sigma}$ are real coefficients with $\sigma(x, t)$ denoting the working subsystem at $(x, t)$.

Take

$$
\begin{aligned}
& T(i, j)=T(i \Delta x, j \Delta t), u(i, j)=u(i \Delta x, j \Delta t), \sigma(i, j)=\sigma(i \Delta x, j \Delta t), \\
& \frac{\partial T(x, t)}{\partial x} \approx \frac{T(i, j)-T(i-1, j)}{\Delta x}, \frac{\partial T(x, t)}{\partial t} \approx \frac{T(i, j+1)-T(i, j)}{\Delta t}
\end{aligned}
$$


Denote $x^{h}(i, j)=T(i-1, j), x^{v}(i, j)=T(i, j)$, where $T(i, j)=T(i \Delta x, j \Delta t)$. It is easy to verify that Eq. (41) can be converted into a $2 \mathrm{D}$ Roesser model (1) with

$$
A^{\sigma(i, j)}=\left[\begin{array}{cc}
0 & 1 \\
\frac{\Delta t}{\Delta x} & 1-\frac{\Delta t}{\Delta x}-a_{0}^{\sigma(i . j)} \Delta t
\end{array}\right], A_{d}^{\sigma(i, j)}=\left[\begin{array}{cc}
0 & 0 \\
0 & -a_{1}^{\sigma(i, j)} \Delta t
\end{array}\right], \quad B^{\sigma(i, j)}=\left[\begin{array}{c}
0 \\
b^{\sigma(i . j)} \Delta t
\end{array}\right],
$$

Let $\Delta t=0.1, \Delta t \leqslant \tau \leqslant 2 \Delta t, \Delta x=0.4, a_{0}^{1}=2.5, a_{0}^{2}=5, a_{1}^{1}=-5, a_{1}^{2}=-2.5, b^{1}=2.5$ and $b^{2}=5$. The thermal process is modeled in the form (1) with

$$
\begin{aligned}
& A^{1}=\left[\begin{array}{cc}
0 & 1 \\
0.25 & 0.5
\end{array}\right], A_{d}^{1}=\left[\begin{array}{cc}
0 & 0 \\
0 & 0.5
\end{array}\right], B^{1}=\left[\begin{array}{c}
0 \\
0.25
\end{array}\right] \\
& A^{2}=\left[\begin{array}{cc}
0 & 1 \\
0.25 & 0.25
\end{array}\right], A_{d}^{2}=\left[\begin{array}{cc}
0 & 0 \\
0 & 0.25
\end{array}\right], B^{2}=\left[\begin{array}{c}
0 \\
0.5
\end{array}\right]
\end{aligned}
$$

Then by using the LMI toolbox and following the steps of Algorithm 1, we make the following records.

Step 1. Solve the LMIs in (36) to obtain $p^{k}, q^{k}, \varsigma_{1}^{k}, \varsigma_{2}^{k}, \zeta^{k}$ and $\psi^{k}$ with $k=1,2$.

$$
\begin{gathered}
p^{1}=\left[\begin{array}{l}
0.7660 \\
0.3613
\end{array}\right], p^{2}=\left[\begin{array}{l}
1.0881 \\
0.5748
\end{array}\right], q^{1}=\left[\begin{array}{l}
0.1813 \\
0.6627
\end{array}\right], q^{2}=\left[\begin{array}{l}
0.3274 \\
0.4545
\end{array}\right], \\
\zeta^{1}=\left[\begin{array}{l}
0.1654 \\
3.5834
\end{array}\right], \zeta^{2}=\left[\begin{array}{l}
0.2728 \\
2.4282
\end{array}\right], \varsigma_{1}^{1}=\left[\begin{array}{l}
0.5383 \\
0.1439
\end{array}\right], \varsigma_{1}^{2}=\left[\begin{array}{l}
0.5935 \\
0.3605
\end{array}\right], \\
\varsigma_{2}^{1}=\left[\begin{array}{l}
0.0405 \\
1.8798
\end{array}\right], \varsigma_{2}^{2}=\left[\begin{array}{l}
0.1065 \\
1.4698
\end{array}\right], \psi^{1}=\left[\begin{array}{l}
0.1004 \\
0.1248
\end{array}\right], \psi^{2}=\left[\begin{array}{l}
0.1879 \\
0.2602
\end{array}\right],
\end{gathered}
$$

Step 2. By $\psi^{k}=K^{k T} B^{k T}\left(p^{k}+\alpha^{-1} q^{k}+D_{H}^{2} \varsigma_{2}^{k}\right)$, compute $K^{k}$ with $k=1,2$.

$$
K^{1}=\left[\begin{array}{ll}
0.0464 & 0.0576
\end{array}\right], K^{2}=\left[\begin{array}{ll}
0.1075 & 0.1489
\end{array}\right] .
$$

Step 3. Compute $\mu$ and $\tau_{a}^{*}$ by (7c) and (7b).

$$
\mu=2.6290, \tau_{a}^{*}=5.9477 \text {. }
$$

Step 4. The desired positive state feedback controller can be given as (34) with the obtained $K^{1}$ and $K^{2}$.

The boundary conditions are given by

$$
\begin{aligned}
& x^{h}(i, j)=0.1, \forall 0 \leqslant j \leqslant 52,-d_{h H} \leqslant i \leqslant 0 ; \\
& x^{v}(i, j)=0.1, \forall 0 \leqslant i \leqslant 52,-d_{v H} \leqslant j \leqslant 0 .
\end{aligned}
$$

Choosing $\tau_{a}=6.12$, the simulation results in Figs. 1 and 2 show the state responses of the resulting closed-loop system under the switching sequence depicted in Fig. 3. It can be observed that the closed-loop system is positive and exponentially stable, which demonstrates the effectiveness of the proposed method.

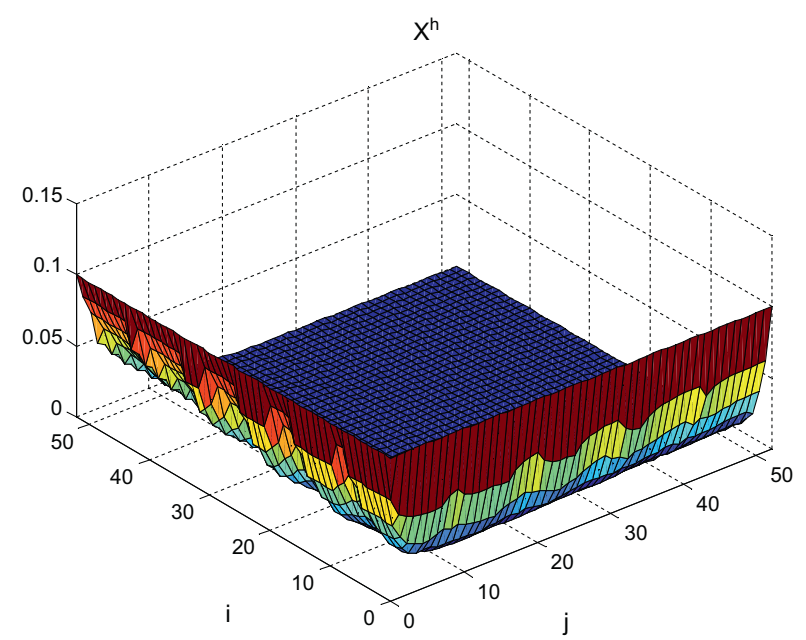

Fig. 1. State response of $x^{h}(i, j)$. 


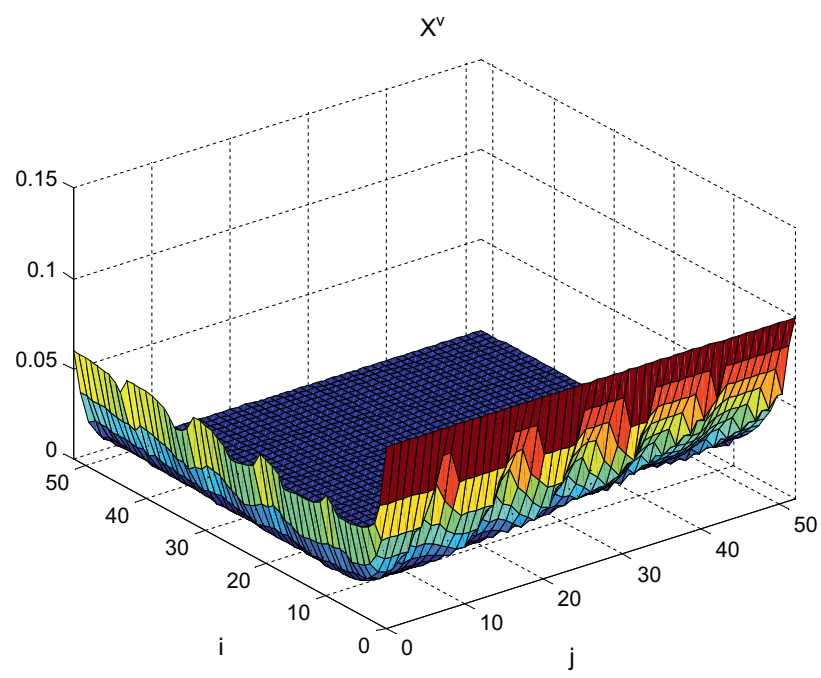

Fig. 2. State response of $x^{v}(i, j)$.

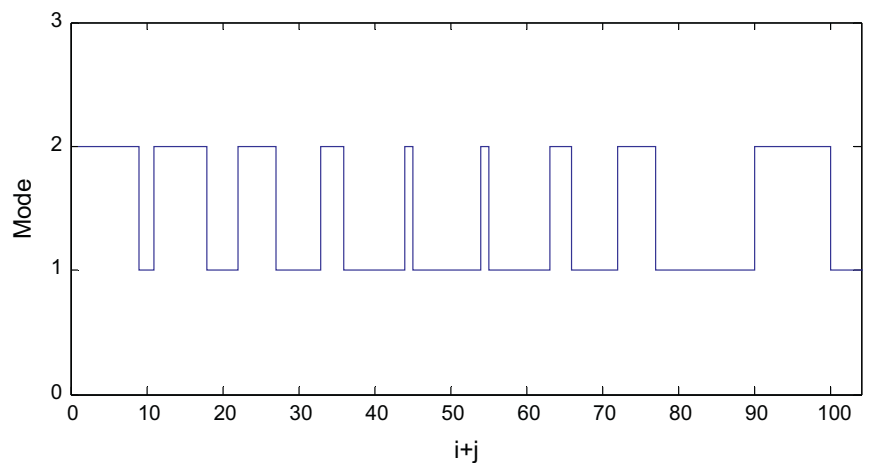

Fig. 3. Switching sequence.

\section{Conclusions}

This paper has addressed the delay-dependent exponential stability analysis and stabilization for positive 2D switched delay systems described by the Roesser model. Sufficient conditions for the delay-dependent exponential stability of positive 2D switched linear systems with delays have been established. A co-positive type Lyapunov functional has been used to get a computationally tractable LMI-based sufficient criterion which ensures the exponential stability. A design methodology of positive feedback controller has been provided to ensure the exponential stability and positivity of the resulting closed-loop system. A numerical example has been given to illustrate the efficiency of the proposed approach. Furthermore, future work will be devoted to the robust control problem to achieve the delay-dependent stability and the disturbance attenuation performance of positive 2D switched systems.

\section{Acknowledgments}

This work was supported by the National Natural Science Foundation of China under Grant No. 61273120 and the Alexander von Humboldt Foundation in Germany.

\section{References}

[1] S.B. Attia, S. Salhi, M. Ksouri, Static switched output feedback stabilization for linear discrete-time switched systems, Int. J. Innov. Comput. Inf. Control 8 (5A) (2012) 3203-3213.

[2] A. Balluchi, M.D. Benedetto, C. Pinello, C. Rossi, A. Sangiovanni-Vincentelli, Cut-off in engine control: a hybrid system approach, in: Proceedings of 36th IEEE Conference on Decision and Control, San Diego, CA, USA, IEEE Press, 1997, pp. 4720-4725.

[3] L. Benvenuti, A. Santis, L. Farina, Positive Systems, Lecture Notes in Control and Information Sciences, Springer-Verlag, Berlin, Germany, 2003. 
[4] A. Benzaouia, A. Hmamed, F. Tadeo, Stability conditions for discrete 2D switched systems based on a multiple Lyapunov function, in: European Control Conference, 2009, pp. 23-26.

[5] A. Benzaouia, A. Hmamed, F. Tadeo, A.E. Hajjaji, Stabilization of discrete 2D time switched systems by state feedback control, Int. J. Syst. Sci. 42 (3) (2011) 479-487.

[6] B.E. Bishop, A.U. Naval, M.D. Annapolis, M.W. Spong, Control of redundant manipulators using logic-based switching, in: Proceedings of 36th IEEE Conference on Decision and Control, 16-18 December 1998, pp. 1488-1493.

[7] M. Bisiacco, New results in 2D optimal control theory, Multidimensional Systems and Signal Processing 6 (3) (1995) $189-222$.

[8] R.N. Bracewell, Two-Dimensional Imaging, Prentice-Hall Signal Processing Series, Prentice-Hall, Englewood Cliffs, NJ, 1995.

[9] M.S. Branicky, Multiple Lyapunov functions and other analysis tools for switched and hybrid systems, IEEE Trans. Automat. Control 43 (4) (1998) 475482 .

[10] B. Castillo-Toledo, S.D. Gennaro, A.G. Loukianov, J. Rivera, Hybrid control of induction motors via sampled closed representations, IEEE Trans. Ind. Electron. 55 (10) (2008) 3758-3771.

[11] R. Cimochowski, Asymptotic stability of the positive switched 2D linear systems described by the Roesser models, in: Proceedings of the 16th International Conference on Methods and Models in Automation and Robotics (MMAR), Miedzyzdroje, 22-25 August 2011, pp. 402-406.

[12] D. Ding, X. Li, Y. Yin, X. Xie, Further studies on relaxed stabilization conditions for discrete-time two-dimension Takagi-Sugeno fuzzy systems, Inf. Sci. 189 (2012) 143-154.

[13] L. Farina, S. Rinaldi, Positive Linear Systems: Theory and Applications, John Wiley \& Sons, Inc., New York, 2000.

[14] E. Fornasini, G. Marchesini, State-space realization theory of two-dimensional filters, IEEE Trans. Automat. Control 21 (4) (1976) 484-491.

[15] E. Fornasini, G. Marchesini, Doubly indexed dynamical systems: state-space models and structural properties, Math. Syst. Theory 12 (1) (1978) 59-72.

[16] J.K. Hale, S.M. Verduyn Lunel, Introduction to Functional Differential Equations, Springer-Verlag, New York, 1993.

[17] T. Hinamoto, Stability of 2-D discrete systems described by the Fornasini-Marchesini second model, IEEE Trans. Circ. Syst.I: Fund. Theory Appl. 44 (3) (1997) 254-257.

[18] S. Huang, Z. Xiang, Delay-dependent stability for discrete 2D switched systems with state delays in the Roesser model, Circ. Syst. Signal Process. (2013), http://dx.doi.org/10.1007/s00034-013-9600-9.

[19] T. Kaczorek, Two-dimensional linear systems, Lecture Notes in Control and Information Sciences, vol. 68, Springer, Berlin, 1985.

[20] T. Kaczorek, Reachability and controllability of non-negative 2D Roesser type models, Bull. Polish Sci.: Tech. Sci. 44 (4) (1996) 405-410.

[21] T. Kaczorek, Positive 1D and 2D Systems, Springer-Verlag, London, 2001.

[22] T. Kaczorek, Realization problem for positive 2D systems with delays, Mach. Intell. Robot. Control 6 (2) (2004) 61-68.

[23] T. Kaczorek, The choice of the forms of Lyapunov functions for positive 2D Roesser model, Int. J. Appl. Math. Comput. Sci. 17 (4) (2007) 471-475.

[24] T. Kaczorek, Asymptotic stability of positive fractional 2D linear systems, Bull. Polish Acad. Sci.: Tech. Sci. 57 (3) (2009) $289-292$.

[25] T. Kaczorek, Asymptotic stability of positive 2D linear systems with delays, Bull. Polish Acad. Sci.: Techn. Sci. 57 (2) (2009) 133-138.

[26] T. Kaczorek, Independence of asymptotic stability of positive 2D linear systems with delays of their delays, Int. J. Appl. Math. Comput. Sci. 19 (2) (2009) 255-261.

[27] J. Kurek, The general state-space model for two-dimensional linear digital systems, IEEE Trans. Automat. Control 30 (6) (1985) 600-602.

[28] X. Li, Z. Xiang, H.R. Karimi, Asynchronously switched control of discrete impulsive switched systems with time delays, Inf. Sci. 249 (2013) $132-142$.

[29] J. Lian, Y. Ge, Robust $H_{\infty}$ output tracking control for switched systems under asynchronous switching, Nonlinear Anal.: Hybrid Syst. 8 (2013) 57-68.

[30] J. Lian, Y. Ge, M. Han, Stabilization for switched stochastic neutral systems under asynchronous switching, Inf. Sci. 222 (2013) 501-508.

[31] J. Lian, C. Mu, P. Shi, Asynchronous $H_{\infty}$ filtering for switched stochastic systems with time-varying delay, Inf. Sci. 224 (2013) $200-212$.

[32] J. Lian, P. Shi, Z. Feng, Passivity and passification for a class of uncertain switched stochastic time-delay systems, IEEE Trans. Cybernet. 43 (1) (2013) 313.

[33] J. Lian, F. Zhang, P. Shi, Sliding mode control of uncertain stochastic hybrid delay systems with average dwell time, Circ. Syst. Signal Process. 31 (2) (2012) 539-553.

[34] H.R. Shaker, R. Wisniewski, Model reduction of switched systems based on switching generalized gramians, Int. J. Innov. Comput. Inf. Control 8 (7B) (2012) 5025-5044.

[35] C. Sreekumar, V. Agarwal, A hybrid control algorithm for voltage regulation in DC-DC boost converter, IEEE Trans. Ind. Electron. 55 (6) (2008) 25302538.

[36] X. Su, P. Shi, L. Wu, S.K. Nguang, Induced $l_{2}$ filtering of fuzzy stochastic systems with time-varying delays, IEEE Trans. Cybernet. 43 (4) (2013) $1251-1264$.

[37] X. Su, P. Shi, L. Wu, Y.D. Song, A novel approach to filter design for T-S fuzzy discrete-time systems with time-varying delay, IEEE Trans. Fuzzy Syst. 20 (6) (2012) 1114-1129.

[38] X. Su, L. Wu, P. Shi, Senor networks with random link failures: distributed filtering for T-S fuzzy Systems, IEEE Trans. Ind. Inf. 9 (3) (2013) 1739-1750.

[39] D. Wang, P. Shi, J. Wang, W. Wang, Delay-dependent exponential $H_{\infty}$ filtering for discrete-time switched delay systems, Int. J. Robust Nonlinear Control $22(13)(2012) 1522-1536$.

[40] R. Wang, Z.G. Wu, P. Shi, Dynamic output feedback control for a class of switched delay systems under asynchronous switching, Inf. Sci. 225 (2013) $72-$ 80.

[41] L. Wu, Z. Feng, W.X. Zheng, Exponential stability analysis for delayed neural networks with switching parameters: average dwell time approach, IEEE Trans. Neural Networks 21 (9) (2010) 1396-1407.

[42] L. Wu, P. Shi, H. Gao, C. Wang, $H_{\infty}$ filtering for 2D Markovian jump systems, Automatica 44 (7) (2008) 1849-1858.

[43] L. Wu, X. Su, P. Shi, J. Qiu, Model approximation for discrete-time state-delay systems in the T-S fuzzy framework, IEEE Trans. Fuzzy Syst. 19 (2) (2011) 366-378.

[44] L. Wu, X. Yao, W.X. Zheng, Generalized $\mathrm{H}_{2}$ fault detection for two-dimensional Markovian jump systems, Automatica 48 (8) (2012) 1741-1750.

[45] L. Wu, W.X. Zheng, Weighted $H_{\infty}$ model reduction for linear switched systems with time-varying delay, Automatica 45 (1) (2009) $186-193$.

[46] L. Wu, W.X. Zheng, H. Gao, Dissipativity-based sliding mode control of switched stochastic systems, IEEE Trans. Automat. Control 58 (3) (2013) 785791.

[47] Z. Xiang, S. Huang, Stability analysis and stabilization of discrete-time 2D switched systems, Circ. Syst. Signal Process. 32 (1) (2013) 401-414.

[48] L. Xu, M. Yamada, Z.P. Lin, O. Saito, Y. Anazawa, Further improvements on Bose's 2D stability test, Int. J. Control Automat. Syst. 2 (3) (2004) 319-332.

[49] G. Zhang, C. Han, Y. Guan, L. Wu, Exponential stability analysis and stabilization of discrete-time nonlinear switched systems with time delays, Int. J. Innov. Comput. Inf. Control 8 (3A) (2012) 1973-1986. 